\title{
Physiologie de la production de colostrum chez la truie
}

\author{
N. DEVILLERS ${ }^{1}$, J. LE DIVIDICH ${ }^{2}$, A. PRUNIER ${ }^{2}$ \\ 1 Agriculture et Agroalimentaire Canada, Centre de Recherche et Développement sur le Bovin Laitier et le Porc, \\ Lennoxville, Québec, J1M 1Z3, Canada \\ 2 INRA, UMR Système d'Élevage et Nutrition Animale et Humaine, 35590 Saint-Gilles \\ Courriel :devillersn@agr.gc.ca
}

Dans l'espèce porcine, la mortalité avant sevrage (13\% des porcelets nés vivants) est très élevée et ne diminue plus depuis une vingtaine d'années. Ces porcelets sont le plus souvent caractérisés par une perte de poids au cours du premier jour de vie indiquant une consommation insuffisante de colostrum. Une meilleure compréhension des mécanismes de sa sécrétion et des facteurs de variation de sa production sont essentiels.

\section{Le Colostrum : définition et composition}

Le colostrum correspond aux premières secrétions de la mamelle dans les heures précédant et suivant la mise bas. Chez la truie, sa composition évolue rapidement au cours des 12 premières heures suivant le début de la mise bas pour devenir progressivement celle du lait au bout de 24 à 36 heures. Le colostrum se caractérise par sa richesse en immunoglobulines. La diminution importante au cours des 24 premières heures du taux de matière sèche de 20-25\% à 18-20\% reflète la chute du taux de protéines, et principalement d'immunoglobulines (figure 1, voir également Darragh et Moughan 1998, Xu 2003). Les composants du colostrum dont les concentrations sont les plus variables sont les lipides et les immunoglobulines. Le taux de lipides dépend principalement de l'alimentation de la truie, alors que les facteurs déterminant le taux d'immunoglobulines n'ont pas encore été clairement identifiés (Rooke et Bland 2002, Farmer et al 2006).

Le porcelet naît dépourvu de protection immunitaire (Bland et al 2003), avec des réserves énergétiques faibles (Herpin et Le Dividich 1995) et sans tissu adipeux brun ni pelage pour maintenir sa température corporelle (Trayhurn et al 1989). Le colostrum produit par la truie au cours des premiè-
Figure 1. Evolution de la teneur des principaux composants du colostrum (Klobasa et al 1987).

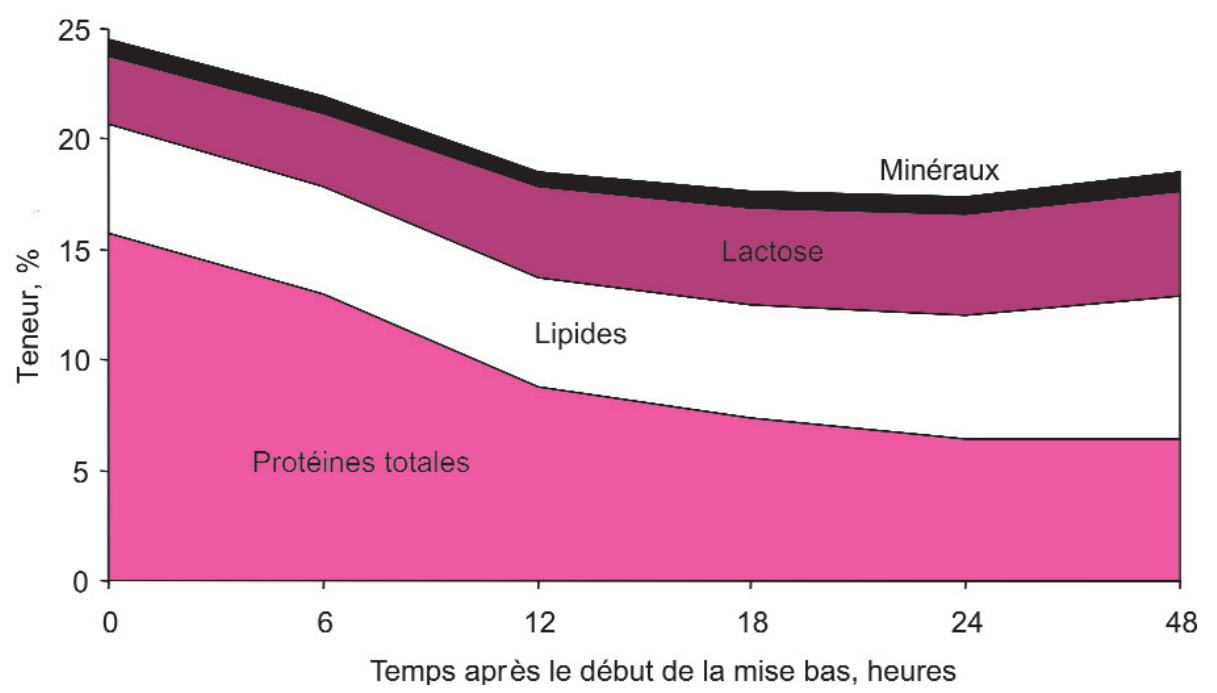

res 24 heures post-partum joue un rôle déterminant pour la survie des porcelets en leur fournissant une protection immunitaire (Rooke et Bland 2002) et suffisamment d'énergie pour leur thermorégulation et leur croissance (Noblet et Le Dividich 1981). La consommation de colostrum par le porcelet et sa production par la truie ont fait l'objet de peu de travaux en conditions d'élevage alors que dans le cadre d'études relativement complexes (alimentation contrôlée), les différents rôles nutritionnel, immunitaire et intestinal du colostrum chez le porcelet ont été déterminés (Xu et al 2000, Rooke et Bland 2002, Le Dividich et al 2005). La réduction de la mortalité précoce des porcelets implique l'identification des facteurs qui déterminent la consommation de colostrum par le porcelet et la production de colostrum par la truie. La consommation de colostrum du porcelet dépend principalement de son poids et de sa vitalité (Devillers et al 2005), mais aussi de la capacité de 
Figure 2. Structure d'une cellule épithéliale mammaire et mécanismes de sécrétion des constituants du colostrum. 1- Exocytose ; 2- Sécrétion des gouttelettes lipidiques ; 3-Voie trans-cellulaire ; 4- Voie para-cellulaire (adapté de Delouis et al 2001).



production de la truie qui n'est pas fonction de la taille de la portée et peut donc être limitante (Le Dividich et al 2004, Devillers et al 2005). La quantité de colostrum produite par une truie varie entre 2 et $5 \mathrm{~kg}$ au cours des premières 24 heures suivant la mise bas (Devillers et al 2005). Cette variabilité de la production de colostrum n'est pas ou peu expliquée par les facteurs zootechniques comme le poids de la truie ou la parité (Devillers et al 2005). Le colostrum est produit aux alentours de la parturition et sa synthèse est sous l'influence et le contrôle de multiples facteurs physiologiques impliqués dans la régulation de la gestation, de la mise bas et de la lactation. La connaissance de la cascade des événements hormo- naux qui surviennent autour de la mise bas et des effets des différentes hormones sexuelles et lactogènes sur la sécrétion de colostrum devrait donc permettre de mieux comprendre l'origine de la variabilité de la production de colostrum chez la truie.

\section{1 / Mécanismes de sécrétion du colostrum}

La lactogenèse correspond à la mise en place de la lactation. Elle commence en fin de gestation et se poursuit jusqu'à l'établissement d'une lactation abondante, c'est-à-dire 24 à 48 heures après le début de la mise bas. La lacto- genèse peut être divisée en deux phases (Hartmann et Holmes 1989) :

- la lactogenèse I est la mise en place du métabolisme mammaire en fin de gestation avec une activité sécrétrice limitée des cellules épithéliales mammaires puis une accumulation progressive de pré-colostrum dans la lumière des alvéoles.

- la lactogenèse II est la mise en place d'une sécrétion abondante par les cellules épithéliales mammaires.

La synthèse de colostrum a lieu essentiellement pendant la lactogenèse I, le colostrum commençant à être synthétisé avant la mise bas (Kensinger et al 1982, 1986). 


\section{1 / Évolution des cellules épi- théliales mammaires en fin de gestation}

A partir de 90 jours de gestation, le développement structural de la mamelle est terminé (Kensinger et al 1982) et l'activité de synthèse et de sécrétion des cellules épithéliales commence à se mettre en place. L'augmentation rapide du ratio ARN/ADN dans la mamelle indique le début de la synthèse des protéines du colostrum par les cellules épithéliales mammaires (Kensinger et al 1982). En parallèle, on observe une accumulation de sécrétions, composées principalement de protéines et de lipides dans la lumière des alvéoles et de gouttelettes lipidiques dans les cellules sécrétrices (Kensinger et al 1982, 1986). En fin de gestation, une grande quantité de gouttelettes lipidiques est présente dans le cytoplasme des cellules sécrétrices ainsi que de nombreuses mitochondries et des vésicules contenant des protéines (Kensinger et al 1986). Les cellules épithéliales mammaires sont donc pleinement opérationnelles en fin de gestation et ont commencé la synthèse des différents éléments du colostrum. A ce stade, 2 à 3 jours avant la mise bas, du colostrum peut déjà être extrait manuellement des tétines (Hartmann et Holmes 1989). A partir de la mise bas, le colostrum contenu dans la mamelle est ingéré par les porcelets, les sécrétions mammaires augmentent et le colostrum est progressivement remplacé par le lait (Kensinger et al 1986).

\section{2 / Sécrétion des composants du colostrum}

Les différents éléments du colostrum sont sécrétés dans la lumière des alvéoles selon quatre voies différentes (figure 2) :

L'exocytose : Elle concerne les éléments contenus dans les vésicules de sécrétion, c'est-à-dire les protéines et le lactose synthétisés dans le réticulum endoplasmique et l'appareil de Golgi (Klopfenstein et al 2002). Ces vésicules contiennent également des électrolytes monovalents $(\mathrm{Na}, \mathrm{K}, \mathrm{Cl})$. Les vésicules de sécrétion cheminent jusqu'à la partie apicale des cellules et fusionnent avec la membrane cellulaire, libérant, par exocytose, leur contenu dans la lumière des alvéoles.

La sécrétion des gouttelettes lipidiques : les gouttelettes lipidiques contenues dans le cytoplasme, après fusion et migration vers la partie apica- le des cellules et enveloppement par la membrane cellulaire apicale sont libérées sous la forme d'un globule lipidique dans la lumière alvéolaire (Keenan 2001).

Le passage trans-cellulaire : Il concerne les immunoglobulines (Ig) provenant du plasma de la mère (Klopfenstein et al 2002) et de nombreux facteurs de croissance et hormones. Le mécanisme d'entrée des Ig depuis les vaisseaux sanguins dans les cellules n'est pas encore complètement élucidé chez la truie. Il met probablement en jeu des récepteurs membranaires au niveau de la partie basale des cellules, comme cela a été montré chez la souris ou la vache (Gitlin et al 1976, Larson et al 1980, Solari et Kraehenbuhl 1984). Les Ig traversent ensuite la membrane cellulaire apicale des cellules pour se retrouver dans la lumière alvéolaire.

Le passage para-cellulaire : au cours de la gestation, les jonctions serrées, qui assurent l'étanchéité de l'épithélium mammaire, sont ouvertes et permettent le passage, entre les cellules épithéliales, des cellules immunitaires, des Ig plasmatiques et des électrolytes vers la lumière alvéolaire (Klopfenstein et al 2002).

Les trois premières voies de sécrétion sont les mêmes pendant la phase colostrale et la phase lactée, alors que la voie para-cellulaire est spécifique de la sécrétion du colostrum. En effet, un des évènements physiologiques marquant le passage de la phase colostrale à la phase lactée est la fermeture des jonctions serrées entre les cellules épithéliales mammaires (Neville et al 2001). Les étapes de la lactogenèse peuvent ainsi être suivies au travers des concentrations plasmatiques de produits spécifiquement synthétisés par la glande mammaire, comme le lactose (Hartmann et al 1984, Atwood et al 1995 , Kent et al 1998), l' $\alpha$-lactalbumine $(\boldsymbol{\alpha}-\mathbf{L} \mathbf{A})$, protéine impliquée dans la synthèse du lactose, ou encore la $\beta$-lactoglobuline ( $\beta$-LG) (Dodd et al 1994) (figure 3 ). Ces produits passent de la lumière alvéolaire dans le plasma par la voie para-cellulaire. Ainsi, dès 5 semaines avant la mise bas, le taux plasmatique élevé de $\beta-\mathrm{LG}$ indique le début de l'expression de certaines protéines du lait (Dodd et al 1994). A partir de 72 à 48 heures avant la mise bas, les concentrations plasmatiques de lactose et d' $\alpha$-LA augmentent, reflétant le début de l'activité sécrétrice des cellules épithéliales (Hartmann et al 1984, Dodd et al 1994). Durant les 24 heures suivant le début de la mise bas, les concentrations plasmatiques de lactose et d' $\alpha$-LA continuent d'augmenter en parallèle avec la concentration de lactose dans le colostrum puis forment un pic et chutent fortement dans les 24 heures suivantes (Hartmann et al 1984, Dodd et al 1994, Devillers et al 2004). La hausse des concentrations de lactose et d' $\alpha$-LA dans le plasma reflète l'augmentation de l'activité de synthèse des cellules épithéliales (Faulkner et al 1982) et plus spécifiquement de la lactose synthétase (Dodd et al 1994). La concentration plasmatique de lactose est corrélée avec celle mesurée dans le colostrum (Hartmann et al 1984, Devillers et al 2004). La chute des concentrations de lactose, d' $\alpha-$ LA et de $\beta-$ LG et la réduction du passage de ces éléments depuis la lumière des alvéoles vers le plasma sont dues à la fermeture des jonctions serrées.

Figure 3. Evolution des concentrations plasmatiques de lactose, $\alpha$-lactalbumine (LA), $\beta$-lactoglobuline (LG) et immunoglobulines $G(\mathrm{lgG})$ chez la truie autour de la parturition (Dodd et al 1994, Hartmann et al 1984, Huang et al 1992).

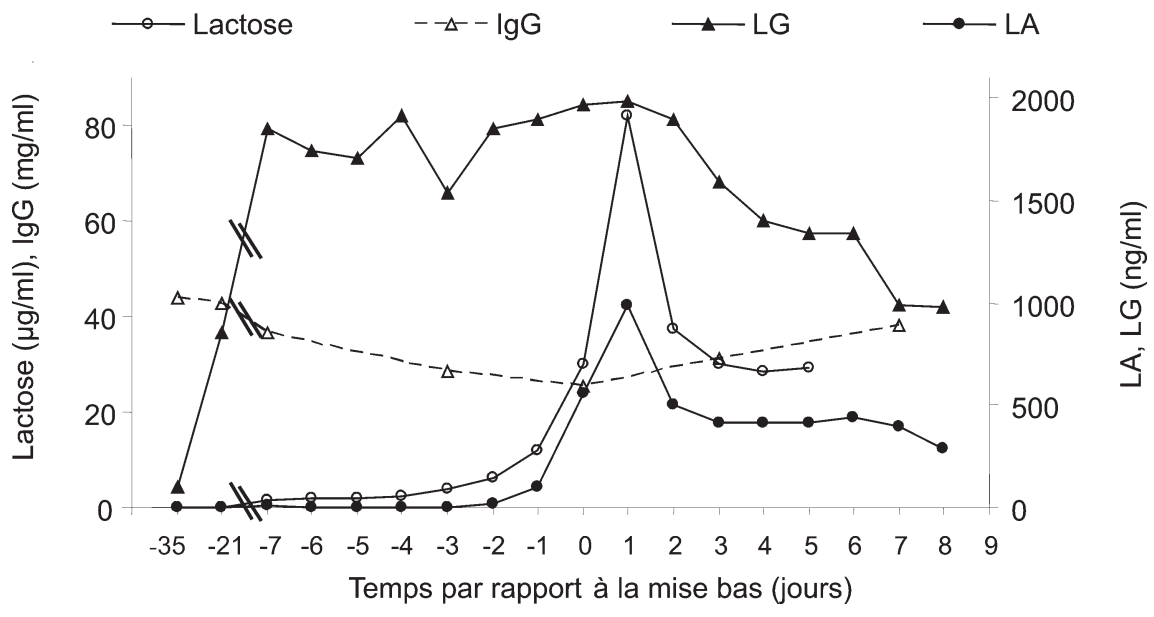


En fin de gestation, la concentration d'Ig dans le plasma diminue en même temps que la concentration plasmatique de lactose augmente (Devillers et al 2004, figure 3 ). Le retour, après la mise bas, des concentrations plasmatiques d'Ig à leur niveau basal est concomitant à la chute de leurs concentrations dans le colostrum et à la fermeture des jonctions serrées. La chute du taux d'Ig dans le colostrum en début de lactation est donc due, au moins en partie, à la fermeture de la voie para-cellulaire.

Les mécanismes de transfert des molécules du plasma vers le colostrum ne sont pas encore complètement élucidés. En effet, la théorie selon laquelle le colostrum serait un transsudat du plasma (Bourne et Curtis 1973), avec un passage libre des molécules par la voie para-cellulaire (Klopfenstein et al 2002), ne permet pas d'expliquer le déséquilibre des concentrations de certaines molécules, comme le glucose, entre le colostrum et le plasma (Atwood et al 1995). Il y aurait donc une régulation de ce transfert, notamment par un passage sélectif de certaines molécules comme cela a été démontré chez la souris (Gitlin et al 1976), la vache (Larson et al 1980) et même chez la truie pour les différentes classes d'IgG (Huang et al 1992). Les concentrations plasmatiques de lactose, $d^{\prime} \alpha-L A$ et de $\beta-L G$ restent à un niveau relativement élevé pendant la lactation par rapport à leur niveau de gestation (figure 3) ce qui montre qu'il subsiste un passage de ces molécules dans le plasma même après la fermeture des jonctions serrées (Dodd et al 1994, Atwood et al 1995). Par conséquent, l'étanchéité de l'épithélium mammaire n'est pas complète, et il existe une autre voie de passage vers le plasma que la voie para-cellulaire.

L'évolution progressive des concentrations des différents composants du colostrum s'explique à la fois par l'évolution de l'activité de synthèse des cellules épithéliales mammaires et par le passage des constituants depuis ou vers le plasma. Ces deux phénomènes sont sous le contrôle de différentes hormones sexuelles et/ou lactogènes.

\section{2 / Endocrinologie de la parturition}

La parturition est la conséquence d'une série de variations hormonales qui aboutissent également à la mise en place de la lactation. Les hormones impliquées sont nombreuses. Elles appartiennent à plusieurs familles et sont sécrétés par des organes très différents comme l'hypophyse, les ovaires et l'utérus.

La cascade d'événements hormonaux aboutissant à la parturition commence vers 72 heures avant la mise bas. En même temps qu'une chute de la concentration plasmatique de progestérone $(\mathbf{P 4})$, on observe une hausse des concentrations de l'oestradiol-17 $\beta$ (E2), du métabolite de la prostaglandine-F2 $\alpha$ (PGF2 $\alpha)$, de la relaxine, du cortisol, de l'ocytocine et de la prolactine (PRL) (Robertson et King 1974,

Figure 4. Evolution des concentrations plasmatiques de progestérone $(P 4)^{3}$, d'oestradiol (E2) ${ }^{3}$, du métabolite de la prostaglandine-F2 $\alpha$ (PGF-M $)^{1}$, de relaxine $(R L X)^{1}$, du cortisol 3 , d'ocytocine $(O T)^{2}$ et de prolactine $(P R L)^{3}$ chez la truie autour de la parturition ( 1 Whitely et al 1990, 2 Castrén et al 1993a et b, 3 Devillers et al 2004).
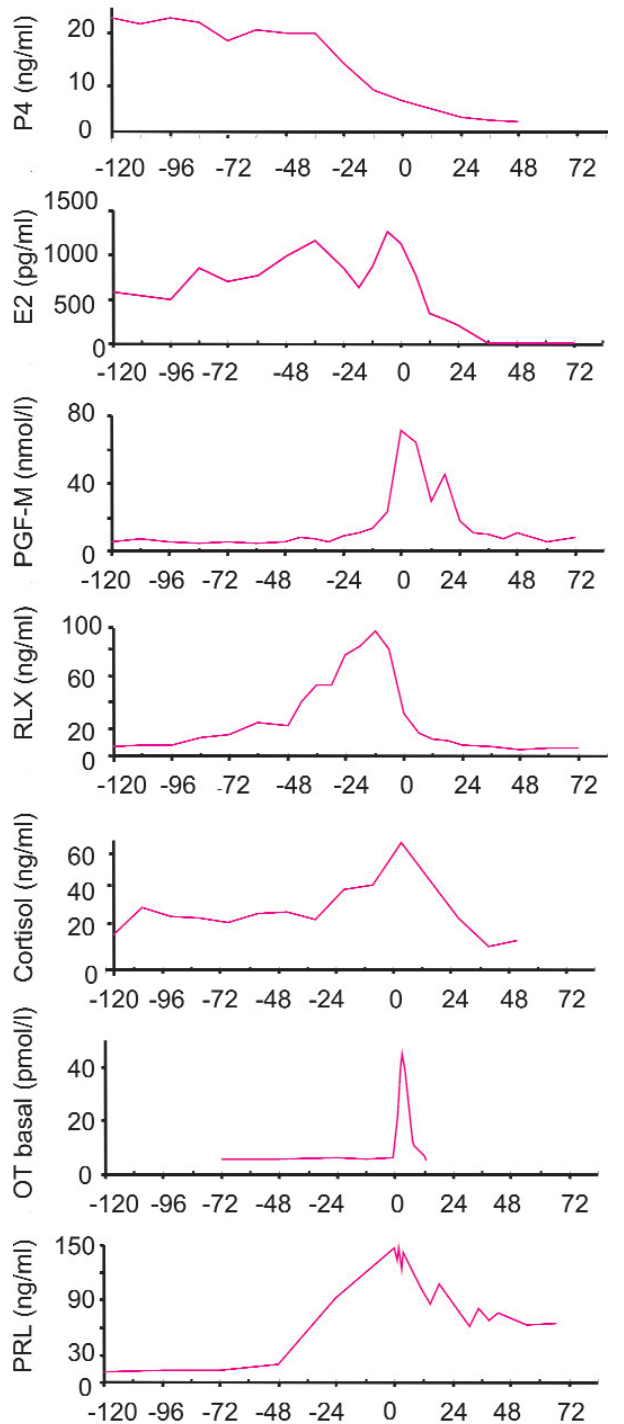

Heure par rapport au début de la mise bas
Forsling et al 1979, Sherwood 1982 , Taverne et al 1982, Whitely et al 1990, Castrén et al 1993a, Jarvis et al 2001, Devillers et al 2004, figure 4).

Le Cortisol foetal : Le signal initial du déclenchement de la parturition proviendrait des fœtus dont les glandes surrénales, arrivant à maturation en fin de gestation, sécrètent des quantités croissantes de cortisol (Silver et al 1979, Randall 1983).

La prostaglandine $F 2 \alpha$ : La sécrétion de PGF2 $\alpha$ par l'utérus serait le signal secondaire du déclenchement de la parturition. En effet, l'administration de PGF $2 \alpha$ en fin de gestation chez la truie induit la mise bas dans les 48 heures qui suivent (Robertson et al 1978). Les prostaglandines exercent une action lutéolytique sur les corps jaunes, sites de production de la P4 (Coggins et al 1977), ce qui entraîne une chute de la concentration plasmatique de $\mathrm{P} 4$ dans les 48 heures précédant la mise bas (Baldwin et Stabenfeldt 1975). Il a aussi été clairement démontré que la PGF2 $\alpha$ stimule la sécrétion d'ocytocine (Ellendorff et al 1979), de prolactine (Taverne et al 1982, Peter et al 1985) et de relaxine (Sherwood 1982, King et Wathes 1989).

La progestérone : Avec la chute de sa concentration plasmatique, le frein exercé par la P4 sur l'action stimulatrice des autres hormones sur la mise bas est levé progressivement. En effet, l'administration de P4 en fin de gestation retarde la mise bas (Gooneratne et al 1979, Whitely et al 1990).

La relaxine : La concentration de relaxine dans le plasma augmente rapidement à partir de 2-3 jours avant la mise bas, atteint un pic dans les 24 heures précédant la mise bas puis chute avant la naissance du premier porcelet (Felder et al 1986, Dlamini et al 1995). Chez la rate, la relaxine stimule la sécrétion basale d'ocytocine (Summerlee et al 1998).

L'oestradiol-17 $\beta$ : $\mathrm{Au}$ cours des trois derniers jours de gestation, la concentration d'E2 augmente et atteint un plateau (Robertson et King 1974, Baldwin et Stabenfeldt 1975). Par la suite, juste avant le début de la mise bas, on observe une chute rapide de la concentration (Robertson et King 1974, Taverne et al 1982, Devillers et al 2004). Pendant la mise bas, la concentration d'E2 continue de chuter très fortement pour devenir non détectable après la délivrance qui par l'évacuation 
des placentas supprime la principale source de synthèse des oestrogènes (Baldwin et Stabenfeldt 1975).

Le cortisol maternel : La concentration de cortisol dans le plasma de la truie commence à augmenter au cours du dernier jour de gestation et atteint un pic centré sur la mise bas (Whitely et al 1984, Meunier-Salaün et al 1991, Devillers et al 2004). La concentration de cortisol retourne par la suite à ses valeurs basales dans les 48 heures suivant la mise bas. Cette élévation du taux de cortisol au cours de la mise bas pourrait être la conséquence du stress induit par la parturition (Molokwu et Wagner 1973) mais également par la contention en cage de mise bas qui empêche l'expression du comportement de nidification (Jarvis et al 2001, 2002). Le cortisol maternel ne serait pas impliqué dans le déclenchement de la mise bas (Killian et al 1973, Ellendorff et al 1979) mais plutôt dans la régulation de l'initiation de la lactation (Whitely et al 1984, Smith et al 1991). Le rôle du cortisol maternel dans le déroulement de la mise bas et les raisons de l'élévation de sa concentration plasmatique chez la truie ne sont donc pas clairement élucidés.

L'ocytocine : L'ocytocine est une hormone à sécrétion pulsatile. Sa concentration en fin de gestation est à la limite du seuil de détection (Forsling et al 1979). Elle augmente fortement dans les heures précédant la mise bas (Castrén et al 1993b, Gilbert 1999) lorsque la concentration de P4 devient inférieure à $10 \mathrm{ng} / \mathrm{ml}$ (Forsling et al 1979), puis redescend en fin de mise bas. L'ocytocine stimulant les contractions utérines, la hauteur de son pic à la mise bas est directement en rapport avec la durée de la mise bas (Castrén et al 1993a). Par ailleurs, la sécrétion d'ocytocine est stimulée par le passage des fœetus dans le col de l'utérus (Gilbert et al 1994). L'ocytocine stimule la contraction des cellules myoépithéliales mammaires ce qui explique une sécrétion et une éjection de colostrum quasi-permanente pendant la mise bas (Smith et al 1991).

La prolactine : La concentration de PRL augmente en fin de gestation à partir de 3 jours avant la mise bas, forme un pic centré sur la mise bas et redescend progressivement tout en restant nettement supérieure au niveau de gestation (Dusza et Krzymowska 1981, Castrén et al 1993b, Devillers et al 2004). La hauteur du pic de PRL à la mise bas et la concentration en début de lactation sont très variables entre les truies avec des valeurs comprises entre 35 et $180 \mathrm{ng} / \mathrm{ml}$ et entre 15 et $135 \mathrm{ng} / \mathrm{ml}$, respectivement (Dusza et Krzymowska 1981, Meunier-Salaün et al 1991, Castrén et al 1993b, de Passillé et al 1993, Dlamini et al 1995, Devillers et al 2004). L'administration de bromocriptine, un inhibiteur de la sécrétion hypophysaire de PRL, n'a aucun effet sur le déroulement de la mise bas ni sur l'évolution des concentrations des autres hormones (Taverne et al 1982). Par contre, la bromocriptine inhibe complètement la lactogenèse (Whitacre et Threlfall 1981, Taverne et al 1982, Farmer et al 1998).

\section{3 / Régulation hormonale de la sécrétion de colostrum}

Les hormones sexuelles et lactogènes influencent la production de colostrum de différentes manières. En premier lieu, elles régulent le développement mammaire pendant la gestation. Par ailleurs, elles contrôlent la synthèse des différents constituants du colostrum. Enfin, elles induisent la fermeture des jonctions serrées entre les cellules épithéliales mammaires et donc le passage de la sécrétion colostrale à la sécrétion lactée. Ceci est particulièrement important car, plus l'évolution de la composition du colostrum, sous l'action des hormones, sera rapide, plus la durée de la période de sécrétion du colostrum sera courte et plus les quantités sécrétées de colostrum et notamment d'Ig seront faibles.

\section{1 / Régulation de la mammo- genèse}

La régulation du développement $\mathrm{du}$ tissu sécréteur de la mamelle est particulièrement importante car le nombre de cellules sécrétrices et le poids de la glande mammaire déterminent en partie son potentiel de sécrétion (Forsyth 1986, Nielsen et al 2001). Pendant la gestation, la croissance de la mamelle est sous l'influence de différentes hormones. La teneur élevée en progestérone et la hausse progressive des concentrations plasmatiques d'oestradiol-17 $\beta$ à partir de 70-80 jours de gestation favorisent la mammogenèse (Forsyth 1986).

L'ovariectomie induit un retard dans le développement lobulo-alvéolaire alors que l'ablation des corps jaunes n'a aucune influence (Buttle 1988). La substance sécrétée par les follicules ou le stroma ovarien, et nécessaire à la croissance mammaire, pourrait être la relaxine (Bagnell et al 1993) qui en association avec l'oestradiol stimule la croissance du parenchyme et des lobules mammaires chez des cochettes ovariectomisées (Winn et al 1994). Dans cette dernière étude, l'association de la progestérone et de la relaxine entraîne la désorganisation de la matrice extracellulaire de collagène, favorisant ainsi la croissance lobulo-alvéolaire. La nécessité de la relaxine pour un développement complet de la glande mammaire a été confirmée chez la truie gestante ovariectomisée (Hurley et al 1991, Zaleski et al 1996).

L'administration d'un antagoniste de la prolactine (bromocryptine ou ergocryptine) au cours du dernier tiers de la gestation (Farmer et al 2000), ou juste pendant les dix derniers jours (Whitacre et Threlfall 1981) inhibe le développement du tissu sécréteur des glandes mammaires. La prolactine est donc nécessaire à la mammogenèse (Farmer 2001). Enfin, les glucocorticoïdes ont une action stimulatrice sur la croissance lobulo-alvéolaire chez la brebis (Delouis et al 1980), mais cet effet n'a pas encore été démontré chez la truie.

\section{2 / Régulation de la synthèse des constituants du colostrum}

Pendant l'initiation de la lactation, c'est-à-dire en fin de gestation et dans les 48 heures suivant la mise bas, les concentrations plasmatiques de P4, d'E2, de relaxine, de cortisol, de PRL et de PGF2 $\alpha$ évoluent rapidement (cf partie 2). Ces hormones agissent sur la synthèse des constituants du colostrum et donc sur l'évolution de sa composition.

La prolactine : L'hormone la plus importante dans la mise en place de la lactation est la PRL. En effet, après administration en fin de gestation de bromocriptine qui inhibe la sécrétion de PRL, la lactation est complètement inhibée (Whitacre et Threlfall 1981, Taverne et al 1982, Farmer et al 1998). De plus, les truies atteintes d'agalactie ont des concentrations plasmatiques de PRL faibles (Threlfall et al 1974). La PRL est donc indispensable à la mise en place de la lactation. En revanche, l'administration de PRL à des truies n'augmente pas leur production laitière (Farmer et al 1999), voire la réduit chez des truies rationnées en protéines (King et al 1996). La prolactine agit sur la production laitière en stimulant la synthèse des caséines, de l' $\alpha-\mathrm{LA}$, du lactose et des acides gras (Plaut et al 1989, Delouis et al 2001). 
La Progestérone : La chute de la $\mathrm{P} 4$ en fin de gestation semble être le facteur déclenchant la lactogenèse. Alors que la P4 stimule la croissance des glandes mammaires (Farmer 2001), elle inhibe la synthèse des constituants du colostrum et du lait. Les concentrations de P4 dans le plasma ou le colostrum et de lactose dans le colostrum sont d'ailleurs négativement corrélées (Martin et al 1978, Willcox et al 1983, Devillers 2004) et l'administration de P4 en fin de gestation retarde l'augmentation de la concentration de lactose dans le colostrum (Gooneratne et al 1979, Whitely et al 1990). L'activité de synthèse des cellules épithéliales mammaires serait donc dépendante de la chute du taux de P4 plasmatique d'une part, et de la hausse des concentrations de PRL d'autre part (Falconer 1980).

D'une manière générale les actions de la P4 et de la PRL s'opposent. Chez la vache, l'action stimulatrice de la PRL sur la synthèse est accrue par le cortisol (Houdebine et al 1985, Forsyth 1986), mais contrecarrée par la P4 (Houdebine et al 1985), ces deux hormones agissant au niveau des récepteurs à la PRL dans la glande mammaire (Houdebine et al 1985). Chez la rate, la P4 inhibe la sécrétion de PRL alors que E2 la stimule (Nicholas et Hartmann 1981). Chez la truie, les liens entre la sécrétion de PRL et les autres hormones ne sont pas clairs. Nous avons vu que la PGF2 $\alpha$ stimule la sécrétion de PRL et d'ocytocine (Ellendorff et al 1979, Taverne et al 1982). Par ailleurs, l'administration de P4 en fin de gestation n'empêche pas le pic de PRL alors qu'elle retarde la mise bas et la lactation (Taverne et al 1982). Enfin, des niveaux élevés de P4 ou un retard dans la chute de la $\mathrm{P} 4$ à la mise bas sont associés avec une hypogalactie (Liptrap 1980, de Passillé et al 1993). Chez la truie, la $\mathrm{P} 4$ agirait donc plutôt sur la glande mammaire (inhibition de l'action de la PRL et de la synthèse des constituants du colostrum) comme chez la vache que sur la sécrétion de la PRL comme chez la rate.

L'oestradiol-17 $\beta$ et les glucocorticoüdes : D'autres hormones modulent l'action de la PRL. L'oestradiol-17 $\beta$ ne participerait pas directement à l'initiation de la lactation chez la truie (Willcox et al 1983), mais l'administration d'E2 augmente la quantité de récepteurs à la PRL dans la glande mammaire chez la rate (Tucker 1981) et augmenterait également la quantité de récepteurs à l'ocytocine chez la brebis (Delouis et al 1980). La hausse des concentrations d'E2 en fin de gestation serait nécessaire à l'initiation de la lactation bien que son rôle exact ne soit pas encore déterminé (Tucker 1981, Willcox et al 1983). De même, chez les ruminants, les glucocorticoïdes augmentent la quantité de récepteurs à la PRL dans la glande mammaire (Delouis et al 1980, Tucker 1981) et potentialisent son action sur les cellules épithéliales mammaires (Delouis et al 1980, Houdebine et al 1985). Ainsi, Willcox et al (1983) trouvent une corrélation positive entre la concentration de cortisol dans le plasma et le taux de lactose dans le colostrum au cours de la mise bas chez la truie, mais cette relation n'est pas retrouvée dans d'autres études (Martin et al 1978, Whitely et al 1990).

La relaxine : La relaxine ne semble pas avoir d'effet particulier sur l'initiation de la lactation chez la truie (Nara et al 1982, Porter et al 1992, Zaleski et al 1996). Cependant, la relaxine inhibe la sécrétion pulsatile d'ocytocine (Summerlee et al 1998), et l'administration de relaxine supprime le réflexe d'éjection du lait chez la rate (Summerlee et al 1984, Jones et Summerlee 1987) mais augmente la sécrétion basale d'ocytocine (Parry et al 1994, Summerlee et al 1998). L'absence d'effet de la relaxine sur la lactation chez la truie pourrait s'expliquer par la concentration basale d'ocytocine très élevée au moment de la parturition, aboutissant à une éjection quasi permanente de colostrum (Lewis et Hurnik 1985). La concentration de relaxine chutant très rapidement avant la fin de la mise bas et avant le retour de la concentration d'ocytocine à des valeurs basses (figure 4), le réflexe d'éjection du colostrum lors de la mise en place des tétées synchronisées n'est pas non plus sous l'influence de la relaxine.

L'ocytocine : Peu d'études ont cherché les facteurs de régulation de l'ocytocine et leur influence sur l'initiation de la lactation chez la truie. On notera l'étude de McDowell et al (1974) qui a montré, in vitro, un effet de la PGF $2 \alpha$ additionnel à celui de l'ocytocine sur la sécrétion de colostrum.

En conclusion, les connaissances sur la régulation de l'initiation de la lactation et de la sécrétion de colostrum chez la truie sont encore réduites et incertaines. L'extrapolation des résultats obtenus sur d'autres mammifères comme les ruminants ou les rongeurs est difficile, l'importance relative de l'action des hormones changeant entre les espèces. D'une manière générale, la chute de la concentration de $\mathrm{P} 4$ et le pic de PRL au moment de la parturition semblent être les principaux évènements initiateurs de la lactation. Une altération dans la coordination des variations de ces deux hormones serait à l'origine des principaux problèmes de lactation, agalactie ou hypogalactie (Einarsson et al 1975, Falconer 1980, Liptrap 1980).

\section{3 / Régulation de la fermeture des jonctions serrées entre les cellules épithéliales mammaires}

Un évènement marquant le passage de la phase colostrale à la phase lactée est la fermeture progressive des jonctions serrées entre les cellules épithéliales mammaires, assurant l'étanchéité des alvéoles par rapport au milieu extracellulaire. La fermeture des jonctions serrées est également sous le contrôle des différentes hormones impliquées dans la régulation de la parturition. Ce contrôle hormonal de la fermeture des jonctions serrées a une incidence sur le passage des $\mathrm{Ig}$ plasmatiques vers le colostrum et donc sur la concentration en $\mathrm{Ig}$ du colostrum.

Si, chez les mammifères, les hormones sexuelles ont une influence directe sur le fonctionnement du système immunitaire (Grossman 1984) et sur la mise en place de la lactation (Falconer 1980), peu d'études ont cherché les effets des différentes hormones sur le transfert des Ig dans le colostrum chez la truie. Chez les ruminants, les changements hormonaux qui ont lieu au moment de la parturition sont fortement impliqués dans la mise en place de la lactation et tout particulièrement dans le transfert des IgG du sérum vers le colostrum (Barrington et al 2001). Ainsi, la chute pre-partum de la progestérone serait le signal déclenchant de la sécrétion du colostrum (Guy et al 1994), et les oestrogènes stimuleraient le passage des $\operatorname{IgG}_{1}$ dans le colostrum chez la vache (Smith 1971, Darton et McDowell 1979). La dexaméthasone, analogue du cortisol, diminue la teneur en $\operatorname{IgG}_{1}$ du colostrum chez la vache (Field et al 1989, Winger et al 1995). De même, la prolactine diminue in vitro l'expression des récepteurs aux $\operatorname{IgG}_{1}$ dans les cellules de la glande mammaire (Barrington et al 1997). In vivo, la prolactine inhibe le transport des $\operatorname{IgG}_{1}$ du sang vers le colostrum et réduit ainsi leur concentration dans le colostrum (Barrington et al 1999). 
Figure 5. Fermeture des jonctions serrées dans la glande mammaire de souris en réponse à l'injection de RU486, antagoniste de la progestérone (P4), ou à une ovariectomie avec ou sans administration de P4. Le saccharose plasmatique représente le niveau de saccharose radiomarqué dans le sang, 5 minutes après son injection dans la lumière alvéolaire. Lorsqu'il est élevé, les jonctions sont ouvertes; la chute du saccharose plasmatique indique l'arrêt du passage du saccharose radiomarqué et donc la fermeture des jonctions (Nguyen et Neville 1998).

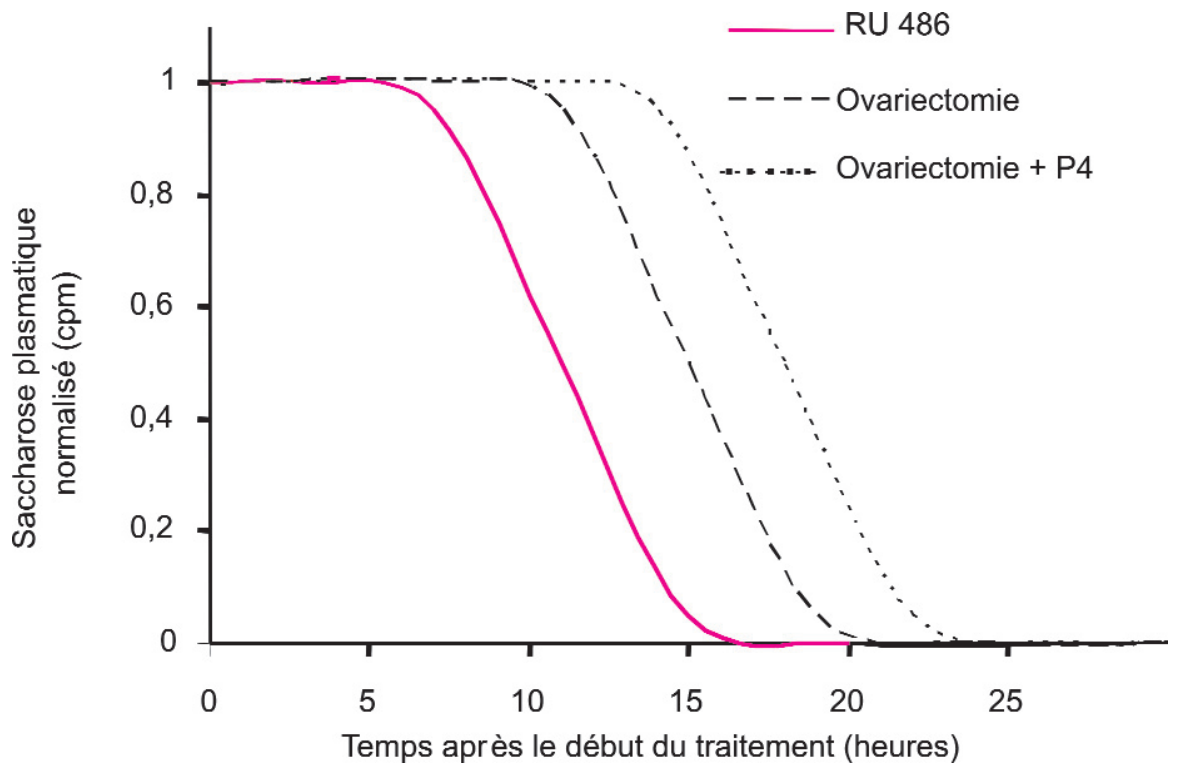

Le contrôle hormonal de la fermeture des jonctions serrées chez la truie a été peu étudié jusqu'à maintenant. Seule, une corrélation négative entre les concentrations d'IgG et de PRL dans le colostrum a été observée (Devillers 2004). Plusieurs études sur l'influence des hormones sur la perméabilité des jonctions serrées ont été réalisées en injectant dans la lumière alvéolaire du saccharose radiomarqué ou de l'albumine fluorescente de manière répétée et en suivant l'évolution de leurs concentrations dans le plasma chez la souris (Nguyen et Neville 1998, Nguyen et al 2001). Il a ainsi été démontré que l'ovariectomie, qui supprime la source de P4, ou l'administration de RU486, antagoniste de la P4, accélère la fermeture des jonctions serrées chez la souris (figure 5). Inversement, l'administration de $\mathrm{P} 4$ après l'ovariectomie retarde cette fermeture (Nguyen et Neville 1998, Nguyen et al 2001).

L'action des glucocorticoïdes a été étudiée chez la vache (Stelwagen et al 1994, 1998, 2000), la chèvre (Thompson 1996) et la souris (Zettl et al 1992, Woo et al 1996, Stelwagen et al 1999) in vitro ou in vivo. Il a ainsi été démontré que le cortisol stimule la formation des jonctions serrées dans la

glande mammaire. Cette action du cortisol peut être contrecarrée par le facteur de croissance TGF- $\beta$ (Woo et al 1996, Nguyen et Neville 1998). La PRL a aussi une action stimulatrice sur la formation des jonctions serrées chez la rate, la souris et la vache (Flint et Gardner 1994, Nguyen et Neville 1998, Barrington et al 2001, Nguyen et al 2001). La présence de PRL ou de cortisol est indispensable à la fermeture des jonctions serrées, et l'action des deux hormones s'additionne (Nguyen et al 2001). Par la suite, la présence de PRL tout au long de la lactation est indispensable pour maintenir l'étanchéité des alvéoles (Flint et Gardner 1994). Enfin, la somatotropine, hormone polypeptidique sécrétée par l'adéno-hypophyse, comme la PRL, n'a aucun effet sur la fermeture des jonctions serrées chez la rate et la vache (Flint et Gardner 1994, Stelwagen et al 1994). En conclusion, ces données montrent, comme pour la régulation de l'activité de synthèse des cellules épithéliales mammaires, que l'initiation de la lactation et la fermeture des jonctions serrées sont induites à la fois par la chute de la P4 en fin de gestation et par l'augmentation des concentrations de PRL et de cortisol au moment de la parturition.

\section{Conclusion}

Si les connaissances sur les mécanismes physiologiques de la sécrétion de colostrum et de sa régulation chez la truie sont encore limitées, l'approche multi-espèces laisse supposer que les changements endocriniens qui ont cours au moment de la parturition influencent la production de colostrum. Les hormones clés sont la prolactine, la progestérone et le cortisol. La progestérone exerce un frein sur l'activité de synthèse des cellules épithéliales mammaires et sur la fermeture de leurs jonctions serrées, alors que la prolactine et le cortisol les stimulent. Le minutage de l'évolution des concentrations de ces hormones autour de la mise bas, ainsi que la balance entre ces hormones déterminent à la fois la quantité de colostrum sécrété via la régulation de l'activité de synthèse des cellules épithéliales mammaires et la période durant laquelle il sera sécrété via l'induction de la fermeture des jonctions serrées. Une altération dans ce minutage ou cette balance pourrait être la cause de désordres dans la production de colostrum.
Sachant que les facteurs environnementaux (logement, température ambiante, lumière, bruit, présence de l'éleveur...) de même que les facteurs endogènes (parité, état sanitaire...) influencent la sécrétion de prolactine et de cortisol, ces facteurs sont susceptibles d'agir indirectement sur la production de colostrum et l'initiation de la lactation. En particulier, il serait intéressant de déterminer dans quelle mesure l'induction de la mise bas, par l'injection de prostaglandines chez la truie, pourrait influencer la production de colostrum en induisant une chute prématurée de la progestérone. De même, une concentration importante de cortisol au moment de la parturition, due à un stress environnemental, serait susceptible d'accélérer la fermeture des jonctions serrées. Les études nécessaires pour vérifier de tels effets sont lourdes à mettre en place mais permettraient de progresser dans la compréhension de l'origine de la variabilité quantitative et qualitative du colostrum, de déterminer quelles sont les conditions favorables à une production optimale de colostrum et in fine d'améliorer la survie des porcelets. 


\section{Références}

Atwood C.S., Toussaint J.K., Hartmann P.E., 1995. Assessment of mammary gland metabolism in the sow. II. Cellular metabolites in the mammary secretion and plasma during lactogenesis. J. Dairy Res., 62, 207-220.

Bagnell C.A., Zhang Q., Downey B., Ainsworth L., 1993. Sources and biological actions of relaxin in pigs. J. Reprod. Fert. Suppl., $48,127-138$.

Baldwin D.M., Stabenfeldt G.H., 1975. Endocrine changes in the pig during late pregnancy, parturition and lactation. Biol. Reprod., $12,508-515$

Barrington G.M., Besser T.E., Gay C.C., Davis W.C., Reeves J.J., McFadden T.B., 1997. Effect of prolactin on in vitro expression of the bovine mammary immunoglobulin G1 receptor. J. Dairy Sci., 80, 94-100.

Barrington G.M., Besser T.E., Gay C.C., Davis W.C., Reeves J.J., McFadden T.B., Akers R.M., 1999. Regulation of the immunoglobulin G1 receptor: effect of prolactin on in vivo expression of the bovine mammary immunoglobulin G1 receptor. J. Endocrinol., 163, 25-31.

Barrington G.M., Mcfadden K., Huyler M.T. Besser T.E., 2001. Regulation of colostrogenesis in cattle. Livest. Prod. Sci., 70, 95-104.

Bland I.M., Rooke J.A., Bland V.C., Sinclair A.G., Edwards S.A., 2003. Appearance of immunoglobulin $\mathrm{G}$ in the plasma of piglets following intake of colostrum, with or without a delay in sucking. Anim. Sci., 77, 277-286.

Bourne F.J., Curtis J., 1973. The transfer of immunoglobins IgG, IgA and IgM from serum to colostrum and milk in the sow. Immunology, 24, 157-162.

Buttle H.L., 1988. Role of the ovaries in inducing mammogenesis in pregnant pigs. J. Endocrinol., 118, 41-45.

Castrén H., Algers B., de Passillé A.M., Rushen J., Uvnas-Moberg K., 1993a. Early milk ejection, prolonged parturition and periparturient oxytocin release in the pig. Anim. Prod., 57, 465471.

Castrén H., Algers B., de Passille A.M., Rushen J., Uvnas-Moberg K., 1993 b. Preparturient variation in progesterone, prolactin, oxytocin and somatostatin in relation to nest building in sows. Appl. Anim. Behav. Sci., $38,91-102$.

Coggins E.G., Van Horn D., First N.L., 1977. Influence of prostaglandin F2 alpha, dexamethasone, progesterone and induced corpora lutea on porcine parturition. J. Anim. Sci., 45, 754-762.

Darragh A.J., Moughan P.J., 1998. The composition of colostrum and milk. In: Verstegen M.W.A., Moughan P.J., Schrama J.W. (Eds), The lactating sow, Wageningen Press, Wageningen, Netherlands, 3-21.

Darton P.J., McDowell G.H., 1979. The effect of oestrogen on selective transfer of IgG1 into mammary secretion of ewes. Aust. J. Exp. Biol. Med. Sci., 57, 617-625.

de Passillé A.M., Rushen J., Foxcroft G.R., Aherne F.X., Schaefer A., 1993. Performance of young pigs: relationships with periparturient progesterone, prolactin, and insulin of sows. J. Anim. Sci., 71, 179-184.

Delouis C., Dijiane J., Houdebine L.M.,
Terqui M., 1980. Relation between hormones and mammary gland function. J. Dairy Sci., 63, 1492-1513.

Delouis C., Houdebine L.M., Richard P., 2001. La lactation. In: Thibault C., Levasseur M.C. (Eds), La reproduction chez les mammifères et l'homme, INRA Editions - Ellipses, Paris, 580-610.

Devillers N., 2004. Variabilité de la production de colostrum chez la truie. Origine et conséquences pour la survie du porcelet. Thèse de Doctorat en Biologie de l'Université de Rennes 1, France, 179p.

Devillers N., Farmer C., Mounier A.M., Le Dividich J., Prunier A., 2004. Hormones, IgG and lactose changes around parturition in plas$\mathrm{ma}$, and colostrum or saliva of multiparous sows. Reprod. Nutr. Dev., 44, 381-396.

Devillers N., Le Dividich J., Farmer C., Mounier A.M., Lefebvre M., Prunier A., 2005. Origine et conséquences de la variabilité de la production de colostrum par la truie et de la consommation de colostrum par les porcelets. Journ. Rech. Porcine Fr., 37, 435-442.

Dlamini B.J., Li Y., Klindt J., Anderson L.L., 1995. Acute shifts in relaxin, progesterone, prolactin, and growth hormone secretion in Chinese Meishan gilts during late pregnancy and after hysterectomy. J. Anim. Sci., 73, 3732-3742.

Dodd S.C., Forsyth I.A., Buttle H.L., Gurr M.I., Dils R.R., 1994. Milk whey proteins in plasma of sows: variation with physiological state. J. Dairy Res., 61, 21-34.

Dusza L., Krzymowska H., 1981. Plasma prolactin levels in sows during pregnancy, parturition and early lactation. J. Reprod. Fertil., 61, 131-134.

Einarsson S., Gustafsson B., Larsson K. 1975. Prostaglandin induced parturition in swine with some aspects on prevention of the MMA (metritis, mastitis, agalactia) syndrome. Nord. Vet. Med., 27, 429-436.

Ellendorff F., Taverne M., Elsaesser F., Forsling M.L., Parvizi N., Naaktgeboren C., Smidt D., 1979. Endocrinology of parturition in the pig. Anim. Reprod. Sci., 2, 323-334.

Falconer I.R., 1980. Aspects of the biochemistry, physiology and endocrinology of lactation. Aust. J. Biol. Sci., 33, 71-84.

Farmer C., 2001. The role of prolactin for mammogenesis and galactopoiesis in swine. Livest. Prod. Sci., 70, 105-113.

Farmer C., Robert S., Rushen J., 1998. Bromocriptine given orally to periparturient of lactating sows inhibits milk production. J. Anim. Sci., 76, 750-757.

Farmer C., Sorensen M.T., Robert S. Petitclerc D., 1999. Administering exogenous porcine prolactin to lactating sows: milk yield, mammary gland composition, and endocrine and behavioral responses. J. Anim. Sci., 77, 18511859.

Farmer C., Sorensen M.T., Petitclerc D., 2000. Inhibition of prolactin in the last trimester of gestation decreases mammary gland development in gilts. J. Anim. Sci., 78, 1303-1309.

Farmer C., Devillers N., Rooke, J.A., Le Dividich J., 2006. Colostrum production in swine: from the mammary gland to the piglets. Pig News Inf. Sous presse.
Faulkner A., Blatchford D.R., White J.M., Peaker M., 1982. Changes in the concentrations of metabolites in milk at the onset and cessation of lactation in the goat. J. Dairy Res., 49, 399405 .

Felder K.J., Molina J.R., Benoit A.M., Anderson L.L., 1986. Precise timing for peak relaxin and decreased progesterone secretion after hysterectomy in the pig. Endocrinology, $119,1502-1509$.

Field R.W., Bretzlaff K.N., Elmore R.G., Rupp G.P., 1989. Effect of induction of parturition on immunoglobulin content of colostrum and calf serum. Theriogenology, 32, 501-506.

Flint D.J., Gardner M., 1994. Evidence that growth hormone stimulates milk synthesis by direct action on the mammary gland and that prolactin exerts effects on milk secretion by maintenance of mammary deoxyribonucleic acid content and tight junction status. Endocrinology, $135,1119-1124$.

Forsling M.L., Taverne M.A.M., Parvizi N., Elsaesser F., Smidt D., Ellendorff F., 1979. Plasma oxytocin and steroid concentrations during late pregnancy, parturition and lactation in the miniature pig. J. Endocrinol., 82, 61-69.

Forsyth I.A., 1986. Variation among species in the endocrine control of mammary growth and function: the roles of prolactin, growth hormone, and placental lactogen. J. Dairy Sci., 69, 886903.

Gilbert C.L., 1999. Oxytocin secretion and management of parturition in the pig. Reprod. Dom. Anim., 34, 193-200.

Gilbert C.L., Goode J.A., McGrath T.J., 1994. Pulsatile secretion of oxytocin during parturition in the pig: temporal relationship with fetal expulsion. J. Physiol., 475, 129-137.

Gitlin J.D., Gitlin J.I., Gitlin D., 1976 Selective transfer of plasma proteins across mammary gland in lactating mouse. Am. J. Physiol., 230, 1594-1602.

Gooneratne A., Hartmann P.E., McCauley I., Martin C.E., 1979. Control of parturition in the sow using progesterone and prostaglandin. Aust. J. Biol. Sci., 32, 587-595.

Grossman C.J., 1984. Regulation of the immune system by sex steroids. Endocr. Rev., 5, 435-455.

Guy M.A., McFadden T.B., Cockrell D.C., Besser T.E., 1994. Regulation of colostrum formation in beef and dairy cows. J. Dairy Sci., 77, 3002-3007.

Hartmann P.E., Holmes M.A., 1989. Sow lactation. In: Barnett J.L., Hennessy D.P. (Eds), Manipulating pig production II, Australasian Pig Science Association, Werribee, Australia, 72-97.

Hartmann P.E., Whitely J.L., Willcox D.L., 1984. Lactose in plasma during lactogenesis, established lactation and weaning in sows. J. Physiol., 347, 453-463.

Herpin P., Le Dividich J., 1995 Thermoregulation and the environment. In: Varley M.A. (Ed), The neonatal pig - development and survival, $\mathrm{CAB}$ International, Oxon, UK, 57-95.

Houdebine L.M., Djiane J., Dusanter-Fourt I., Martel P., Kelly P.A., Devinoy E., Servely J.L., 1985. Hormonal action controlling mammary activity. J. Dairy Sci., 68, 489-500. 
Huang S.C., Hu Z.L., Hasler-Rapacz J., Rapacz J., 1992. Preferential mammary storage and secretion of immunoglobulin gamma (IgG) subclasses in swine. J. Reprod. Immunol., 21, $15-28$.

Hurley W.L., Doane R.M., O’Day-Bowman M.B., Winn R.J., Mojonnier L.E., Sherwood O.D., 1991. Effect of relaxin on mammary development in ovariectomized pregnant gilts. Endocrinology, 128, 1285-1290.

Jarvis S., van der Vegt B.J., V Lawrence A.B., Mclean K.A., Deans L.A., Chirnside J., Calvert S.K., 2001. The effect of parity and environmental restriction on behavioural and physiological responses of pre-parturient pigs. Appl. Anim. Behav. Sci., 71, 203-216.

Jarvis S., Calvert S.K., Stevenson J.S., Leeuwen N., Lawrence A.B., 2002. Pituitaryadrenal activation in pre-parturient pigs (Sus scrofa) is associated with behavioural restriction due to lack of space rather than nesting substrate. Anim. Welf., 11, 371-384.

Jones S.A., Summerlee A.J., 1987. Effects of chronic infusion of porcine relaxin on oxytocin release in lactating rats. J. Endocrinol., 114, 241246.

Keenan T.W., 2001. Milk lipid globules and their surrounding membrane: a brief history and perspectives of future research. J. Mammary Gland Biol. Neoplasia, 6, 365-371.

Kensinger R.S., Collier R.J., Bazer F.W., Ducsay C.A., Becker H.N., 1982. Nucleic acid, metabolic and histological changes in gilt mammary tissue during pregnancy and lactogenesis. J. Anim. Sci., 54, 1297-1308.

Kensinger R.S., Collier R.J., Bazer F.W., 1986. Ultrastructural changes in porcine mammary tissue during lactogenesis. J. Anat., 145, 49-59.

Kent J.C., Arthur P.G., Hartmann P.E., 1998. Citrate, calcium, phosphate and magnesium in sows' milk at initiation of lactation. J. Dairy Res., 65, 55-68.

Killian D.B., Garverick H.A., Day B.N., 1973. Peripheral plasma progesterone and corticoid levels at parturition in the sow. J. Anim. Sci., 37, 1371-1375.

King G.J., Wathes D.C., 1989. Relaxin, progesterone and estrogen profiles in sow plasma during natural and induced parturitions. Anim. Reprod. Sci., 20, 213-220.

King R.H., Pettigrew J.E., McNamara J.P., McMurtry J.P., Henderson T.L., Hathaway M.R., Sower A.F., 1996. The effect of exogenous prolactin on lactation performance of first litter sows given protein deficient diets during the first pregnancy. Anim. Reprod. Sci., 41, 37-50.

Klobasa F., Werhahn E., Butler J.E., 1987. Composition of sow milk during lactation. J. Anim. Sci., 64, 1458-1466.

Klopfenstein C., Couture Y., Martineau G.P., Bouchard E., 2002. Physiopathologie comparative de la lactation chez la truie et chez la vache. Médecin Véterinaire du Québec, 32, 52-56.

Larson B.L., Heary H.L.J., Devery J.E., 1980. Immunoglobulin production and transport by the mammary gland. J. Dairy Sci., 63, 665-671.

Le Dividich J., Martineau G.P., Thomas F., Demay H., Renoult H., Homo C., Boutin D., Gaillard L., Surel Y., Bouetard R., Massard M., 2004. Acquisition de l'immunité passive chez les porcelets et production de colostrum chez la truie. Journ. Rech. Porcine Fr., 36, 451-456.
Le Dividich J., Rooke J.A., Herpin H., 2005. Nutrirional and immunological importance of colostrum for the new-born pig. J. Agric. Sci., $143,469-485$

Lewis N.J., Hurnik J.F., 1985. The development of nursing behaviour in swine. Appl. Anim. Behav. Sci., 14, 225-232.

Liptrap R.M., 1980. Prostaglandin F2 alpha and progesterone in experimental hypogalactia in sows. Res. Vet. Sci., 29, 240-247.

Martin C.E., Hartmann P.E., Gooneratne A., 1978. Progesterone and corticosteroids in the initiation of lactation in the sow. Aust. J. Biol. Sci., $31,517-525$

McDowell W.S., Mather E.C., Martin C.E., 1974. The effect in vitro of prostaglandins with oxytocin on the milk-ejection activity of lactating sow mammary tissue. J. Reprod. Fertil., 40, 229-233.

Meunier-Salaün M.C., Gort F., Prunier A., Schouten W.P.G., 1991. Behavioural patterns and progesterone, cortisol and prolactin levels around parturition in European (Large-White) and Chinese (Meishan) sows. Appl. Anim. Behav. Sci., 31, 43-59.

Molokwu E.C., Wagner W.X., 1973. Endocrine physiology of the puerperal sow. J. Anim. Sci., 36, 1158-1163.

Nara B.S., Welk F.A., Rutherford J.E. Sherwood O.D., First N.L., 1982. Effect of relaxin on parturition and frequency of live births in pigs. J. Reprod. Fertil., 66, 359-365.

Neville M.C., Morton J., Umemura S., 2001. Lactogenesis. The transition from pregnancy to lactation. Pediatr. Clin. North Am., 48, 35-52.

Nguyen D.A., Neville M.C., 1998. Tight junction regulation in the mammary gland. $\mathrm{J}$. Mammary Gland Biol. Neoplasia, 3, 233-246.

Nguyen D.A., Parlow A.F., Neville M.C., 2001. Hormonal regulation of tight junction closure in the mouse mammary epithelium during the transition from pregnancy to lactation. J. Endocrinol., 170, 347-356.

Nicholas K.R., Hartmann P.E., 1981. Progressive changes in plasma progesterone, prolactin and corticosteroid levels during late pregnancy and the initiation of lactose synthesis in the rat. Aust. J. Biol. Sci., 34, 445-454.

Nielsen O.L., Pedersen A.R., Sorensen M.T., 2001. Relationships between piglet growth rate and mammary gland size of the sow. Livest. Prod. Sci., 67, 273-279.

Noblet J., Le Dividich J., 1981. Energy metabolism of the newborn pig during the first $24 \mathrm{~h}$ or life. Biol. Neonate., 40, 175-182.

Parry L.J., Poterski R.S., Summerlee A.J., 1994. Effects of relaxin on blood pressure and the release of vasopressin and oxytocin in anesthetized rats during pregnancy and lactation. Biol. Reprod., 50, 622-628.

Peter A.T., Huether P., Doble E., Liptrap R.M., 1985. Prostaglandin F2 alpha and prolactin in experimental hypogalactia in sows. Res. Vet. Sci., 39, 222-229.

Plaut K.I., Kensinger R.S., Griel L.C., Kavanaugh J.F., 1989. Relationships among prolactin binding, prolactin concentrations in plasma and metabolic activity of the porcine mammary gland. J. Anim. Sci., 67, 1509-1519.

Porter D.G., Friendship R.M., Ryan P.L., Wasnidge C., 1992. Relaxin is not associated with poor milk yield in the postpartum sow. Can. J. Vet. Res., 56, 204-207.
Randall G.C., 1983. Changes in the concentrations of corticosteroids in the blood of fetal pigs and their dams during late gestation and labor Biol. Reprod., 29, 1077-1084.

Robertson H.A., King G.J., 1974. Plasma concentrations of progesterone, oestrone, oestradiol17 beta and of oestrone sulphate in the pig at implantation, during pregnancy and at parturition. J. Reprod. Fertil., 40, 133-141.

Robertson H.A., King G.J., Elliot J.I., 1978. Control of the time of parturition in sows with prostaglandin F2alpha. Can. J. Comp. Med., 42, 32-34.

Rooke J.A., Bland I.M., 2002. The acquisition of passive immunity in the new-born piglet. Livest. Prod. Sci., 78, 13-23.

Sherwood O.D., 1982. Relaxin at parturition in the pig. In: Cole D.J.A., Foxcroft G.R. (Eds), Control of pig reproduction, Butterworth Scientific, London, UK, 343-375.

Silver M., Barnes R.J., Comline R.S., Fowden A.L., Clover L., Mitchell M.D., 1979. Prostaglandins in the foetal pig and prepartum endocrine changes in mother and feotus. Anim. Reprod. Sci., 2, 305-322.

Smith K.L., 1971. Role of estrogen in the selective transport of IgGL into the mammary gland. J. Dairy Sci., 54, 1322-1323.

Smith N.A., Toussaint J.K., Hartmann P.E., 1991. Peaks in the sow's intramammary pressure occur immediatly after rather than either just before or at piglet delivery. In: Batterham E.S. (Ed), Manipulating pig production, Australasian Pig Science Association, Werribee, Australia, 75.

Solari R., Kraehenbuhl J.P., 1984. Biosynthesis of the immunoglobulin A antibody receptor. A model for the transepithelial sorting of a membrane glycoprotein. Cell, 36, 61-72.

Stelwagen K., Davis S.R., Farr V.C., Eichler S.J., Politis I., 1994. Effect of once daily milking and concurrent somatotropin on mammary tight junction permeability and yield of cows. J. Dairy Sci., 77, 2994-3001.

Stelwagen K., van Espen D.C., Verkerk G.A., McFadden H.A., Farr V.C., 1998. Elevated plasma cortisol reduces permeability of mammary tight junctions in the lactating bovine mammary epithelium. J. Endocrinol., 159, 173-178.

Stelwagen K., McFadden H.A., Demmer J., 1999. Prolactin, alone or in combination with glucocorticoids, enhances tight junction formation and expression of the tight junction protein occludin in mammary cells. Mol. Cell Endocrinol., 156, 55-61.

Stelwagen K., Hopster H., van der Werf J.T., Blokhuis H.J., 2000. Short communication: effects of isolation stress on mammary tight junctions in lactating dairy cows. J. Dairy Sci., 83, 48-51.

Summerlee A.J., O'Byrne K.T., Paisley A.C., Breeze M.F., Porter D.G., 1984. Relaxin affects the central control of oxytocin release. Nature, $309,372-374$

Summerlee A.J., O’Byrne K.T., Poterski R.S., 1998. Relaxin inhibits the pulsatile release of oxytocin but increases basal concentrations of hormone in lactating rats. Biol. Reprod., 58, 977 981.

Taverne M., Bevers M.M., Bradshaw J.M.C., Dieleman S.J., Willemse A.H, Porter D.G, 1982. Plasma concentrations of prolactin, progesterone, relaxin and oestradiol-17b in sows treated with progesterone, bromocryptine or 
indomethacin during late pregnancy. J. Reprod. Fertil., 65, 85-96.

Thompson G.E., 1996. Cortisol and regulation of tight junctions in the mammary gland of the late-pregnant goat. J. Dairy Res., 63, 305-308.

Threlfall W.R., Dale H.E., Martin C.E., 1974. Serum and adenohypophyseal concentrations of prolactin in sows affected with agalactia. Am. J. Vet. Res., 35, 313-315.

Trayhurn P., Temple N.J., Van Aerde J., 1989. Evidence from immunoblotting studies on uncoupling protein that brown adipose tissue is not present in the domestic pig. Can. J. Physiol. Pharmacol., 67, 1480-1485.

Tucker H.A., 1981. Physiological control of mammary growth, lactogenesis, and lactation. J. Dairy Sci., 64, 1403-1421.

Whitacre M.D., Threlfall W.R., 1981. Effects of ergocryptine on plasma prolactin, luteinizing hormone, and progesterone in the periparturient sow. Am. J. Vet. Res., 42, 1538-1538.

Whitely J.L., Willcox D.L., Newton J.A., Bryant-Greenwood G.D., Hartmann P.E., 1984.
Total and free plasma concentrations of progesterone, cortisol and oestradiol-17beta during pregnancy, parturition and early lactation in sows. Aust. J. Biol. Sci., 37, 267-276.

Whitely J.L., Hartmann P.E., Willcox D.L., Bryant-Greenwood G.D., Greenwood F.C., 1990 Initiation of parturition and lactation in the sow: effects of delaying parturition with medroxyprogesterone acetate. J. Endocrinol., 124, 475484.

Willcox D.L., Arthur P.G., Hartmann P.E., Whitely J.L., 1983. Perinatal changes in plasma oestradiol-17beta, cortisol and progesterone and the initiation of lactation in sows. Aust. J. Biol. Sci., 36, 173-181.

Winger K., Gay C.C., Besser T.E., 1995. Immunoglobulin G1 transfer into induced mammary secretions: the effect of dexamethasone. J. Dairy Sci., 78, 1306-1309.

Winn R.J., Baker M.D., Merle C.A., Sherwood O.D., 1994. Individual and combined effects of relaxin, estrogen and progeterone in ovariectomized gilts. II. Effects on mammary development. Endocrinology, 135, 1250-1255.
Woo P.L., Cha H.H., Singer K.L., Firestone G.L., 1996. Antagonistic regulation of tight junction dynamics by glucocorticoids and transforming growth factor-beta in mouse mammary epithelial cells. J. Biol. Chem., 271, 404-412.

Xu R.J., 2000. Postnatal adaptation of the gastrointestinal tract in neonatal pigs: a possible role of milk-borne growth factors. Livestock Prod. Sci., 66, 95-107.

Xu R.J., 2003. Composition of porcine milk. In: Xu R.J., Cranwell P. (Eds), The neonatal pig - gastrointestinal physiology and nutrition, Nottingham University Press, Nottingham, UK, 213-246.

Zaleski H.M., Winn R.J., Jennings R.L., Sherwood O.D. 1996. Effects of relaxin on lactational performance in ovariectomized gilts. Biol. Reprod., 55, 671-675.

Zettl K.S., Sjaastad M.D., Riskin P.M., Parry G., Machen T.E., Firestone G.L., 1992. Glucocorticoid-induced formation of tight junctions in mouse mammary epithelial cells in vitro. Proc. Natl. Acad. Sci. U.S.A., 89, 9069-9073.

\section{Résumé}

Le colostrum joue un rôle indispensable dans la survie du porcelet nouveau-né en lui apportant de l'énergie et une protection immunitaire. La production de colostrum par la truie est très variable et les facteurs l'influençant n'ont pas encore été clairement identifiés. La sécrétion du colostrum intervient autour de la parturition et est susceptible d'être contrôlée par les différentes hormones régulant la gestation, la parturition et l'initiation de la lactation. Chez d'autres espèces, l'influence prépondérante de la prolactine, de la progestérone et du cortisol dans l'initiation de la lactation a déjà été démontrée. L'avancement des connaissances sur la régulation hormonale de la colostrogenèse est une voie prometteuse pour trouver des solutions concrètes en terme de conduite d'élevage qui permettront d'augmenter la production de colostrum de la truie et de réduire la mortalité précoce du porcelet.

\section{Abstract}

\section{Physiology of colostrum production in sows}

Colostrum intake is one of the main factors determining piglets' survival. Colostrum provides to piglet energy and immune protection. However, colostrum production is very variable among sows and factors controlling this production have not been yet clearly identified. Colostrum secretion occurs during parturition and may be influenced by hormones which control pregnancy, parturition and lactation initiation. In other species, the major role of prolactin, progesterone and cortisol in lactation initiation has been yet determined. Understanding the origin of colostrum production variability and hence finding management solutions to increase colostrum production and decrease piglet mortality, may be provided by further research on the endocrine and physiological control of colostrum secretion.

DEVILLERS N., LE DIVIDICH J., PRUNIER A., 2006. Physiologie de la production de colostrum chez la truie. INRA Prod. Anim., 19, 29-38. 